\title{
Violences des jeunes à Lubumbashi avant, pendant et après les élections de décembre 2018
}

\author{
Par LUPITSHI WA NUMBI Norbert* et MUMBA KAKUDJI Martial**
}

\section{INTRODUCTION}

La présente communication s'articule autour des points suivants :

- Esquisse conceptuelle sur la notion de violence;

- survol épistémologique autour de la notion de « jeunesse » et/ou de « jeune »;

- A la découverte de ce que sont les jeunes (acteurs pluriels);

- Actes et comportements de violence observés à Lubumbashi avant, pendant et après les élections du 30 décembre dernier;

- Synthèse interprétative des faits de violence relevés.

\section{A. Esquisse conceptuelle sur la notion de violence}

Sonia HARRATI, David VAVASSORI et Loïck VILLERBU $(2006,11-22)^{1}$ indiquent que la délinquance et la violence ne sont pas des données au contenu clair et précis. Ce sont des constructions sociales résultant d'une sélection de faits et d'une interprétation de ce qui fait problème dans une société donnée, à un moment donné. Si ces deux phénomènes sont universels, ce qui change d'une société à l'autre, d'une époque à l'autre, ce sont:

- les comportements qualifiés de délinquants ou de violents;

- les catégories de personnes considérées comme délinquants ou violents;

- les instances ayant la compétence de sanctionner ces comportements;

- les types de réactions à ces comportements;

- les justifications des sanctions appliquées à ces comportements;

- les conséquences de ces réactions pour l'acteur délinquant ou violent et/ou pour la société.

De façon courante, la violence est présentée laconiquement comme « une force brutale exercée contre quelqu'un ». Une appréhension systématique vient de MICHAUD (cité par les trois auteurs, pp 21-22) qui estime qu'il y a violence quand, " dans une situation d'interaction, un ou plusieurs acteurs agissent de manière directe ou indirecte, massée ou distribuée, en portant atteinte à un ou plusieurs autres, à des degrés variables, soit dans leur inté-

* Professeur à l’Université de Lubumbashi, Docteur en criminologie

** Chef de Travaux à la Faculté de Droit de l'Université de Lubumbashi, DEA en Droit Public et Avocat au Barreau de Lubumbashi.

1 Sonia HARRATI at al. (2006), Délinquance et violence, Paris, Armand Colin,. 
grité morale, soit dans leurs possessions, soit dans leurs participations symboliques et culturelles ».

Partant de cette conception ouverte, on distingue les formes de violence suivantes :

- les violences physiques : elles concernent les atteintes à l'intégrité corporelle (exemple : coups, voies de faits, atteintes et sévices corporels, etc.);

- les violences verbales : elles concernent le contenu des paroles et le mode de communication, de l'intimidation jusqu'aux menaces d'agression physique ou des menaces de rétorsion (par exemple : injures, insultes, dénigrement, etc.);

- violences psychiques : elles concernent les atteintes à l'intégrité psychique ou mentale de l'autre telles que son estime de soi, son identité personnelle (par exemple : menace, harcèlement, humiliation, etc.);

- violences sexuelles (confère loi congolaise sur les violences sexuelles);

- violences matérielles : il s'agit des destructions, de la désappropriation abusive de biens, d'objets investis d'une valeur affective (par exemple; vandalisme, vol, cambriolage, etc.);

- violences économiques et sociales : elles concernent la manipulation financière (par exemple : escroquerie financière, licenciement abusif) et toute autre forme de discrimination (par exemple : discrimination ethnique, culturelle, religieuse, sexuelle, etc.).

\section{B. Petit sursaut épistémologique autour de la notion de « jeunesse » ou de « jeune »}

Les jeunes forment une catégorie de personnes sémantiquement et moralement malheureuse car, faute de définition claire, ils sont victimes des clichés disqualifiants.

Olivier GALLAND $(2011,12-23)^{2}$ relève plusieurs syntagmes et qualifications associés à la jeunesse. Il note que le « jeune » est présenté facilement comme « quelqu'un qui n'a pas encore les qualités de maturité », un personnage infra social, encore teinté d'une animalité qui le conduit facilement à l'indocilité, à la colère, à l'intempérance, à l'impureté... Par conséquent, tout ce qu'il posera comme acte est d'ores et déjà désordonné.

Dans cet angle d'approche, on rattache à la notion de " jeunesse » l'idée d'étourderie, de vivacité, de folie, de débauche, de manque d'expérience, d'emportement de l'âge, d'imprudence, de légèreté, d'impatience, de manque de réflexion, etc.

Si ces clichés péjoratifs restent encore vivaces dans notre conscience, croyez-moi, la société congolaise manquera de neutralité dans l'appréciation objective des actes et comportements imputés aux jeunes.

Nous pensons, pour notre part, que la jeunesse forme juste une catégorie particulière de personnes appelant, en société, « une attention, une empathie, un accompagnement et un encadrement » à cause de la fragilité et de la malléabilité de leur caractère, comme cela est bien explicité dans l'exposé des motifs de la loi numéro 09/001 du 10 janvier 2009 portant protection de l'enfant en RDC.

2 Olivier GALLAND (2011), Sociologie de la jeunesse, Paris, Armand Colin,. 
Plus loin, le même Olivier GALLAND (déjà cité) constate encore que malgré les " écarts » liés à l'âge, la jeunesse, c'est toujours l'âge le plus aimable et le plus brillant de la vie [...] ceux qui parle de la vieillesse, comme sage, mûre et modérée, pour faire rougir la jeunesse, comme vicieuse, folle et débauchée, ne sont pas des justes appréciateurs de la valeur des choses; car les imperfections de la vieillesse sont assurément plus nombreuses et plus incurables que celles de la jeunesse.

NAGELS et REA $(2007,7-9)^{3}$, chercheuses belges soutiennent que la jeunesse est une construction sociale. Il n'existe pas une « jeunesse » au monde mais bien des « jeunesses », selon la place qu'elle occupe dans la société et sa participation aux rapports sociaux.

Il est beau et alléchant d'affirmer combien la jeunesse est le fervent, l'avenir, le tison et l'espoir de toute société mais WALLANT $(1992,9)^{4}$ soutient effectivement que la jeunesse, c'est l'avenir, mais, dit-il encore, " l'avenir commence aujourd'hui et ne peut se préparer qu'au regard du passé avec le meilleur des connaissances disponibles. L'Afrique sera ce que sa jeunesse sera. Réhabilitée, respectée, enseignée correctement, responsabilisée, celle-ci constituera alors l'espoir du continent.

$\mathrm{Vu}$ l'argumentaire qui précède, les jeunes ne peuvent donc être et faire que ce que leur société veut qu'ils soient et fassent.

\section{Les jeunes, acteurs pluriels}

Recelant en eux plusieurs potentialités, notamment la vigueur, la vitalité, l'endurance physique, le dynamisme, le goût de l'aventure et du risque, la témérité, etc. tout cela associé à la malléabilité de leur caractère, les jeunes deviennent des acteurs «pluriels » au comportement tout à fait imprédictible. En un mot, des sujets difficiles à comprendre et à gérer.

En effet, Bernard LAHIRE (1998) $)^{5}$ note que l'acteur pluriel est un sujet qui accumule diverses expériences socialisatrices à cause de son appartenance (à) ou son évolution dans plusieurs structures ou institutions sociales. Chacune de ces expériences socialisatrices influe sur sa personne dans la définition des situations concrètes et dans son agir comportemental. Cet acteur se caractérise par l'imprédictibilité de son comportement, car chaque institution lui communique une expérience qu'il internalise et mobilise dans tel ou tel autre contexte. Dans sa vie, l'acteur pluriel connaît la simultanéité de rôles au point où il devient même difficile de lui coller une seule identité.

WALLANT (déjà cité), fort de son expérience personnelle de plusieurs années de vie et de recherche en Afrique noire, souligne que la jeunesse africaine, majoritaire dans la population, représente une masse énorme exposée aux sollicitations les plus suspectes et aux explosions les plus incontrôlables.

3 NAGELS et Andrea REA (2007), Jeunes à perpète. Génération à problèmes ou problème de génération, Louvain-la-Neuve (Belgique), Academia-Bruylant.

4 WALLANT (1992), Jeunesse marginalisée. Espoir de l'Afrique, Paris, L'Harmattan.

5 Bernard LAHIRE (1998), L'homme pluriel, Paris, l'Harmattan. 
En 2005, les chercheurs de l'OCU ${ }^{6}$ mettaient déjà en exergue les rapports mitigés que les jeunes de la rue entretenaient avec les autorités politico-administratives locales. Ils ont fait remarquer que ces jeunes se mettent ou sont souvent mis à l'avant-plan dans diverses manifestations politiques (marches pacifiques ou de protestation, campagnes électorales, meetings, etc.). Ils sont surtout mobilisés par diverses forces sociales comme " meilleurs » perturbateurs de certaines manifestations dont on ne veut pas l'organisation ou la réussite; ils sont également réquisitionnés dans des milices pour y apporter de la vivacité que la société leur reconnait.

Pour sa part, KAUMBA LUFUNDA $(2007,74)^{7}$ constatait, après les élections de 2006, que les jeunes désœuvrés de nos villes (congolaises), les enfants soldats et les démobilisés de l'armée, les enfants de la rue ${ }^{8}$ en quête d'une activité valorisante, constituaient un réservoir tout indiqué pour les agents de recrutement et de conscription.

Considérant le fait que, toute proportion gardée, les mêmes causes produisent les mêmes effets, il n'est donc pas surprenant de constater aujourd'hui que les jeunes gens soient encore impliqués dans les violences qui ont émaillé les élections de 2018.

Cela étant dit, tâtons du doigt ce qui a été observé sur le terrain.

\section{Violences des jeunes avant, pendant et après les élections du 30 décembre 2018 à Lubumbashi}

Honnêteté scientifique oblige, je dois, de prime abord, signaler que le recueil des faits cidessous narrés s'est réalisé avec le concours circonstanciel des étudiants de Master préparatoire et de Bac 2 de l'Ecole de criminologie de l'Université de Lubumbashi.

\section{Violences avant les élections}

Il importe de noter de prime abord qu'il a toujours existé une violence permanente dans toute communauté humaine. Les violences décrites ne sont donc pas une nouveauté dans la ville ni l'apanage exclusif des élections. Il est toujours observé quotidiennement à Lubumbashi des violences physiques et verbales perpétrées par les jeunes :

- violences physiques : atteinte à l'intégrité physique et à la propriété d'autrui.

A ce registre, on note, entre autres : les bagarres, les coups et blessures, le vol public dans ses diverses variantes (à la tire, à l'arrachée, dans les rayons des magasins, etc.), le cambriolage des établissements commerciaux.

6 OCU : Observatoire du changement urbain de l'Université de Lubumbashi.

7 KAUMBA LUFUNDA (2007), "Les défis de la sécurité pré-électorale et " post-électorale ", Conclave du Mont- AMBA des universitaires congolais, Elections, paix et développement en RDC. Prise de position des universités congolaises, Kinshasa du 13 au 15 septembre 2006, pp. 62-79.

8 Que moi je nomme « jeunes de la rue » dans LUPITSHI WA NUMBI, N. (2013) Sortir de la rue. Les trajectoires des jeunes de Lubumbashi, Louvain-la-Neuve, Academia-Harmattan, 
Par rapport aux élections, les six formes de violences déjà évoquées ont été enregistrées : pendant la campagne électorale, les affiches de certains candidats ont été soit enlevées, déchirées, effacées ou dénaturées au moyen de la peinture, surtout lors de l'accueil de certains candidats présidents de la république et cela a été décrié sur les antennes des radios et télévisions locales. Ces actes ont été souvent accompagnés de la violence verbale, toujours à l'endroit de certains candidats non désirés.

- violences verbales : injures publiques à l'endroit de certains candidats et de leurs militants, insanités de tout genre et cela, au vu et au su de tout le monde, commentaires injurieux sur la morphologie et le visage de certains candidats, chants calomnieux et provocateurs à l'endroit de certaines personnalités politiques (par exemple : « MBEMBA akosi MOISE EH! »; « Yo nani, oboya X (le nom de leur candidat préféré!), intimidation et menace, etc.

De façon concrète, voici quelques cas de violence vécus pendant la période de campagne électorale :

1) Au niveau de l'Arrêt de bus EXPRESS, sur Boulevard MSIRI, avant l'arrivée du candidat de LAMUKA aux présidentielles, certains jeunes ont contraint avec menace plusieurs paisibles passants à aller accueillir leur candidat « président de la république ». Ceux qui ont refusé se sont vu brutalisés au vu du public.

2) Sur les avenues MSIRI et LUMUMBA, des jeunes avaient été aperçus entrain d'arracher et de déchirer sans vergogne et sans inquiétude les affiches du candidat président de la république du Front commun pour le Congo (FCC). En outre, ils s'attaquaient aussi à toute personne qui portait le T-shirt à image du candidat FCC. D'autres jeunes s'adonnaient à cœur joie à badigéonner l'image du même candidat de la peinture pour le défigurer.

3) $\mathrm{Au}$ croisement des avenues SENDWE et MOERO dans la commune Lubumbashi, lorsque le cortège accompagnant le candidat de LAMUKA (aux présidentielles) approchait de la poste, une voiture en provenant de la commune de KENIA s'était arrêtée, le chauffeur l'a incendié puis a couru pour se jeter dans la foule.

\section{Violences pendant les élections}

Il faut d'abord souligner que le jour de vote, la campagne était déjà déclarée close. De ce fait, il n'y a pas eu assez de provocation entre militants de différentes formations politiques. Néanmoins, sporadiquement, il a été observé quelque violences verbales et physiques, par exemple : crier fort le nom ou le numéro de son candidat devant le bureau de vote dans l'intention soit de gêner les autres ou d'influencer leur vote. Il y a eu aussi des jeunes gens qui déclaraient anticipativement la victoire de leur candidat et parfois ils le faisaient haut et fort en langue tribale de certains candidats. Il a été aussi observé par-ci et par-là des bousculades dans les files d'attente devant les bureaux de vote. 
On remarquera que c'est beaucoup plus après les élections que les violences se sont révélées plus manifestes.

\section{Violences après les élections}

1. Au quartier Bel air, sur l'avenue Des cimetières, juste après la barrière du bureau de la police, dans l'euphorie de la victoire de leur candidat aux présidentielles, deux filles et un garçon se sont promenés nus et ne se sont rhabillés qu'à l'arrivée des éléments de la police.

2. Toujours au quartier Bel air, sur l'Avenue Des plaines, des jeunes avaient délimité l'espace ou des zones accessibles aux seuls alliés du Candidat de LAMUKA. Par malchance, un paisible citoyen qui voulait passer dans cette zone avec une Jeep couverte des banderoles de PPRD s'est vu arrêté et sa voiture incendiée. En revanche, d'autres jeunes de tendance autre que celle de LAMUKA se sont, à leur tour, organisés pour affronter ceux de LAMUKA. D'après les témoins interrogés, cet affrontement a duré trois jours et la police qui s'est montrée impuissante a dû faire appel à l'armée pour calmer la situation.

3. Au quartier GAMBELA, sur l'Avenue Des conservateurs, un Monsieur non identifié faisant partie de l'équipe de campagne électorale du Candidat de FCC s'est montré, deux jours avant la proclamation des résultats provisoires, très rassuré de la victoire de leur candidat. Il est allé sensibiliser les jeunes dans une parcelle pour leur dire qu'il y aura rencontre festive après victoire impérative du candidat $N^{\circ} 13$. Après proclamation des résultats en défaveur de leur candidat, le Monsieur n'a plus tenu à sa promesse, et il s'est vu tabassé par les jeunes et n'a eu la vie sauve que grâce à l'intervention de la police.

4. Sur Boulevard M'SIRI, entre les Arrêts de bus Texaco et Express, un jeune motard a pris sur sa moto un chien habillé d'un chapeau portant l'image du candidat malheureux de FCC.

5. Sur l'Avenue KALUBWE dans la commune de Lubumbashi, une famille qui attendait la victoire du Candidat de LAMUKA, s'était déjà bien organisée pour la fête préparant boissons et nourritures pour la circonstance. Mais à l'échec de leur candidat, le chef de famille transforma toute la boisson et toute la nourriture de fête en marchandises. Cela choqua tous les membres de sa famille qui s'en prirent à lui. 


\section{E. Quelle compréhension exacte avoir de toutes ces violences perpétrées par les jeunes à Lubumbashi?}

Criminologiquement parlant, les faits de violence narrés peuvent s'interpréter sous quatre registres suivants :

\section{Le registre de l'indolence ou de la mollesse des agences de contrôle social}

Ce registre peut être soutenu par la théorie dite « de la vitre cassée ». Cette théorie montre que la négligence de réparation ou de remplacement d'une vitre cassée sur un bâtiment peut entrainer la casse des autres vitres restantes. Car, à travers cette vitre cassée, les passants croiront en une bâtisse abandonnée dont personne ne s'occupe.

Par analogie, sur le plan criminologique, il est prouvé que lorsque la société tolère régulièrement (impunément) la commission de certaines incivilités, leurs auteurs prennent l'habitude et perçoivent en cela (en cette indolence) la consécration de l'impunité dans la société. Voilà qui explique plausiblement une bonne part de violences permanentes observées quotidiennement dans la ville de Lubumbashi. C'est dans cette optique que s'explique, par exemple, les chants à caractère injurieux entonnés régulièrement lors des cortèges funéraires; c'est aussi le cas des insanités proférées publiquement par les étudiants de l'UNILU lorsqu'ils sont à bord de leur bus de transport.

Nous estimons que la RDC doit cesser de demeurer une société anomique, trop indolente sur le plan sanctionnel.

\section{Le registre de l'affirmation de soi ou d'une identité}

Forts de leur vitalité physique et de leur dynamisme incontesté, les jeunes aiment exhiber à la face du monde leur présence ou leur existence. Ils veulent s'assumer en tant que « sujets historiques " du sociologue français Alain TOURRAINE ou plutôt comme " acteurs sociaux » de Christian DEBUYST. Ils refusent d'être réduits au rang de simples réceptacles ou « idiots culturels » (dont parle GOFFMANN). Ils revendiquent une existence sociale, montent des projets et veulent imposer leurs points de vue, même si de fois la manière de le faire peut se révéler moralement hypothétique. Avons-nous le droit de leur interdire cela? Je ne pense pas.

Nous trouvons, pour notre part, qu'il serait intéressant pour la société d'approcher les jeunes gens et attirer leur attention sur une " affirmation de soi démesurée » aux conséquences fâcheuses pour eux-mêmes, pour leurs proches et pour leur société.

\section{Le registre de la manipulation}

Suite à la malléabilité de leur caractère et de la vulnérabilité de leur position sociale (manque de pouvoir décisionnel), les jeunes constituent, comme l'ont affirmé KAUMBA LUFUNDA et WALLANT (déjà cités), une intéressante " proie » à la solde de toutes sortes 
de sollicitations dont ils ne maitrisent pas toujours les tenants et les aboutissants. Et lorsqu'il y a traque, les jeunes tombent souvent dans les filets de la justice et en paient les frais et parfois, à l'insu ou à l'indifférence notoire de leurs manipulateurs.

\section{Le registre de la provocation}

Les recherches psychologiques attestent que les jeunes rêvent souvent " grand ». Par conséquent, ils veulent être écoutés, valorisés et respectés. C'est ainsi que Paul GRELL (1999, $247)^{9}$ souligne que pour les jeunes, même s'ils vivent dans une condition sociale inconfortable (manque d'avoir et de pouvoir), cela n'implique pas pour eux une faiblesse de caractère ou une lâcheté; bien au contraire, ils souhaitent voir en cela une certaine grandeur enfouie dans des comportements obstinément orientés vers un vouloir vivre, honoré par tous $^{10}$.

C'est donc une entreprise très risquée, pour la société ou pour tout individu, des tenir aux jeunes gens des promesses fallacieuses ou fantaisistes. Ils vous prennent aux mots et vous attendent au tournant.

\section{CONCLUSION}

En conclusion et au regard de quatre registres explicatifs ci-dessus commentés, on peut donc acquiescer avec CROZIER et FRIEDBERG (1997), que « tous les phénomènes sociaux ou toutes les pratiques sociales qu'on observe, si aberrants peuvent-ils paraître de prime abord, ont toujours un sens et correspondent à une rationalité à partir du moment qu'ils existent ${ }^{11}$ ». C'est le cas de la violence perpétrée par les jeunes, avant, pendant et après les élections qu'il sied d'analyser correctement car entourée de plusieurs motivations et enjeux non apparents.

\section{BIBLIOGRAPHIE SELECTIVE}

1. Bernard LAHIRE (1998), L'homme pluriel, Paris, l'Harmattan.

2. CROZIER, M et FRIEDBERG, E. (1997), L'acteur social et le système, Paris, Editions du Seuil.

3. KAUMBA LUFUNDA (2007), "Les défis de la sécurité pré-électorale et " post-électorale ", Conclave du Mont- AMBA des universitaires congolais, Elections, paix et développement en RDC. Prise de position des universités congolaises, Kinshasa du 13 au 15 septembre 2006, pp. $62-$ 79.

4. LUPITSHI WA NUMBI, N. (2013), Sortir de la rue. Les trajectoires des jeunes de Lubumbashi, Louvain-la-Neuve, Academia-Harmattan.

9 Paul GRELL (1999), Les jeunes face à un monde précaire, Paris, L'Harmattan.

10 Ajouté par nous.

11 CROZIER, M et FRIEDBERG, E. (1997), L'acteur social et le système, Paris, Editions du Seuil. 
5. NAGELS et Andrea REA (2007), Jeunes à perpète. Génération à problèmes ou problème de génération, Louvain-la-Neuve (Belgique), Academia-Bruylant.

6. Olivier GALLAND (2011), Sociologie de la jeunesse, Paris, Armand Colin.

7. Paul GRELL (1999), Les jeunes face à un monde précaire, Paris, L'Harmattan.

8. Sonia HARRATI at al. (2006), Délinquance et violence, Paris, Armand Colin.

9. WALLANT (1992), Jeunesse marginalisée. Espoir de l'Afrique, Paris, L'Harmattan. 